\title{
Grupo Focal como metodologia de pesquisa na Residência Médica
}

\section{Luciana Segurado Côrtes ${ }^{1}$, Ana Maria Costa ${ }^{2}$, Antonio Carlos Rodrigues da Cunha ${ }^{3}$ e Letícia Segurado Côrtes ${ }^{4}$}

${ }^{1}$ Programa de Residência Médica de Ginecologia e Obstetrícia do Hospital Regional Leste do Distrito Federal, Brasil | lucianasegurado@yahoo.com.br I https://orcid.org/00000-00028952-08322

${ }^{2}$ Programa de Pós-graduação da Escola Superior de Ciências da Saúde - ESCS/DF, Brasil | dotorana@gmail.com | http://orcid.org/0000-0002-1931-3969

${ }^{3}$ Conselho de Ética em Pesquisa Universidade de Brasília, Brasil I acrc@unb.br I https://orcid.org/0000-0001-6238-3287

${ }^{4}$ Faculdade de Informação e Comunicação Universidade Federal de Goiás, Brasil I leticiascortes@ufg.br I https://orcid.org/0000-0003-0196-4728

Resumo: Introdução: A Residência Médica representa a melhor forma de capacitação em serviço após a conclusão da faculdade de Medicina. A Ginecologia e Obstetrícia possui um órgão regulador nacional denominado de Febrasgo (Federação Brasileira de Ginecologia e Obstetrícia) que desenvolveu uma matriz de competência para uniformizar todos os programas de Residência Médica. Essa mudança despertou o desejo de realizar uma pesquisa com as residentes de um hospital público. Objetivos: O presente estudo resolveu avaliar se o eixo da matriz de competência referente às questões de sexualidades (que representa o décimo eixo), eram contempladas no Programa de Residência Médica de Ginecologia e Obstetrícia do referido hospital. Métodos e Discussão: A metodologia utilizada foi a qualitativa, na modalidade de grupo focal, como forma de avaliar se as profissionais em formação de um hospital público obtiveram contato com pacientes de orientaç̃es sexuais homoafetivas durante o percurso de três anos da Residência. Desse modo, foram reunidas em ambiente neutro e a reunião foi coordenada por um moderador e uma auxiliar de pesquisa. Resultados e Conclusões: A partir do grupo focal, pode-se perceber que um terço das residentes teve contato com o atendimento de pacientes do objeto de estudo e, assim, foram acrescidos à programação teórica tópicos referentes a esse tema, com o intuito de contemplar a matriz de competência.

Palavras-chave: Residência Médica; Matriz de Competência; Grupo focal.

\section{Focus group as teaching strategy in Medical Residency}

Abstract: Introduction: Medical Residency represents the best form of in-service training after completing medical school. Gynecology and Obstetrics has a national regulatory body called Febrasgo (Brazilian Federation of Gynecology and Obstetrics) that has developed a competency matrix to standardize all Medical Residency programs. This change aroused the desire to conduct a survey with the residents of a public hospital. Objectives: The present study decided to assess whether the axis of the competence matrix related to sexuality issues (which represents the tenth axis), were included in the Medical Residency Program of Gynecology and Obstetrics of the referred hospital. Methods and Discussion: The methodology used was qualitative, in the form of a focus group, as a way of assessing whether the professionals in formation at a public hospital had contact with patients of homo-affective sexual orientations during the three-year course of the Residence. Thus, they were brought together in a neutral environment and the meeting was coordinated by a moderator and a research assistant. Results and Conclusions: From the focus group, it can be seen that one third of the residents had contact with the care of patients of the object of study and, thus, topics related to this theme were added to the theoretical program, in order to contemplate the matrix of competence.

Keywords: Medical Residence; Competency Matrix; Focus Group. 


\section{Introdução: Motivações para a Pesquisa}

A Residência Médica (RM) é a melhor forma de capacitação do médico em serviço (Feitosa et al, 2020) e os Programas de RM, que são a modalidade de ensino de pós-graduação destinada a médicos sob a forma de curso de especialização, representam o modelo dominante de formação pós-graduada em medicina nas Américas e Europa e tem, como característica, o vínculo entre o aprendizado e a experiência em serviços de saúde qualificados. Hoje é o alvo quase obrigatório dos estudantes de medicina nos países do 1 ำ Mundo (Romão, 2017).

O modelo atual de RM deriva daquele experimentado por Halsted e articulado por este juntamente com Osler e Welch no Johns Hopkins Hospital, situado em Baltimore, Maryland-EUA, com a finalidade de corrigir as inadequações decorrentes do treinamento não-estruturado e assegurar que o médico residente desenvolva as competências necessárias à prática na sua especialidade de escolha (Long, 2000).

Desse modo, a formação do residente de Obstetrícia e Ginecologia envolve múltiplos aspectos: como o conhecimento clínico, o desenvolvimento de habilidades e técnicas, além do amadurecimento de atitudes pessoais, éticas e profissionais (Júnior, 2007). Assim, os futuros tocoginecologistas dependerão, para uma boa formação profissional, de seu esforço individual e de boas condições de treinamento oferecidas por parte das instituições formadoras, destacando-se a importância essencial da atuação dos preceptores dos programas. $\mathrm{O}$ treinamento durante a residência médica deve propiciar ao médico residente domínio suficiente para ingressar no competitivo mercado de trabalho de modo a permitir o atendimento necessário às suas futuras pacientes, com boa qualidade técnica e humanística (Júnior, 2007).

Assim, os programas de RM têm, como característica principal, o desenvolvimento de conhecimentos, habilidades e atitudes, de forma sistematizada e supervisionada por profissionais competentes, que procuram oferecer a formação em todos os seus aspectos: técnicos, éticos e humanísticos (Romão, 2017).

Como forma de corroborar isso, a Federação Brasileira de Ginecologia e Obstetrícia (Febrasgo) desenvolveu recentemente uma matriz de competências para a formação de médicos residentes $(\mathrm{R})$ em ginecologia e obstetrícia, com base no "The Obstetrics and Gynecology Milestone Project", que foi elaborado pelo American Board of Obstetrics and Gynecology (ABOG) e pelo American College of Obstetrics e Ginecologia (ACOG) (Sá e Romão, 2019). A Matriz de Competências em Ginecologia e Obstetrícia constitui uma importante referência para uniformizar e qualificar os programas de residência médica nessa especialidade em todo Brasil, como também para orientar os processos de formação e avaliação do residente e de certificação do especialista em Ginecologia e Obstetrícia (Sá e Romão, 2019).

Os objetivos essenciais dessa matriz é: assegurar maior consistência e coerência na orientação dos Programas de Residência Médica (PRM) em Ginecologia e Obstetrícia; distribuir as competências de maneira hierarquizada e crescente em complexidade para 0 primeiro, segundo e terceiro ano de residência; referenciar a avaliação do médico residente em Ginecologia e Obstetrícia para cada um dos seus componentes: conhecimentos, habilidades e atitudes; permitir o aprendizado do residente a partir da avaliação transparente e ajustada ao seu nível de progressão; referenciar a avaliação de Programas de Residência Médica em Ginecologia e Obstetrícia e orientar a preceptoria e supervisão local dos Programas de Residência Médica em Ginecologia e Obstetrícia (Febrasgo, 2019).

Ao avaliar o 10ำ eixo, referente à atenção à saúde e cuidados em ginecologia endócrina, observa-se que o residente do $1^{\circ}$ ano deve demonstrar conhecimento básico sobre a resposta sexual feminina e masculina e aspectos biopsicossociais da construção da sexualidade, incluindo aspectos da orientação sexual e identidade de gênero. 
O residente do $2^{\circ}$ ano deve estar habilitado a realizar diagnóstico diferencial, acompanhamento clínico e abordagem terapêutica multiprofissional das disfunções sexuais de origem orgânica (dispareunia, vaginismo, vulvodinia), psíquica e disforia de gênero, para os componentes de Conhecimento $(C)$, Habilidade $(H)$ e Atitude $(A)$ (Febrasgo, 2019).

Desse modo, por ser um tema atual, despertou a intenção de discuti-lo e avaliá-lo na população de residentes do hospital público do Distrito Federal utilizando o grupo focal, como estratégia de ensino nesse processo de formação profissional médica.

\section{Questões Mobilizadoras}

Diante dessa nova mudança de abordagem da Residência Médica sobre a forma de matriz de competências e referente às questões de sexualidade, surgiram inquietações nos pesquisadores para determinar se as profissionais em formação possuíam familiaridade em relação ao tema de homossexualidade e, se o mesmo, havia se descortinado a elas durante essa etapa de crescimento profissional no Programa de Residência Médica de Ginecologia e Obstetrícia de um hospital público.

Assim, esse artigo representou uma parte da dissertação de Mestrado, que foi realizada com os residentes acima referidos, porém foi avaliada somente a vertente relacionada com a pós-graduação médica e homossexualidade, porém na pesquisa completa houve a abordagem da presença do tema da homossexualidade na educação familiar, escolar, formação acadêmica.

\section{Método e Discussão}

Em um grupo de nove residentes (três do primeiro ano; três do segundo e três do terceiro ano, utilizou-se a técnica de um grupo focal (GF) preconizada por Minayo, (2014) e que, de acordo com essa autora, por se tratar de um grupo pequeno e homogêneo pode-se aprofundar o consenso entre os participantes.

Os critérios de inclusão do estudo foram: ser residentes de Ginecologia e Obstetrícia de um hospital público do Distrito Federal, voluntárias e com assinatura de termo de consentimento livre e esclarecido (TCLE) assinado previamente à realização do GF. Já em relação aos critérios de exclusão, foram não ser residentes, não aceitarem a participação na pesquisa e não assinarem o TCLE.

O GF é aplicado como técnica por pesquisador que tem como objetivo coletar informações sobre um determinado tema específico, por meio da discussão participativa entre os participantes, reunidos em um mesmo local e durante certo período (Dall'agnol, 2016). O local no qual será realizado o grupo deve ser idealmente neutro, acessível e silencioso e a duração média de um grupo é de uma hora e trinta minutos (Borges e Santos, 2005).

Segundo Minayo, (2014), a técnica deve ser aplicada mediante um roteiro que vai do geral ao específico, em ambiente não diretivo, sob a coordenação de um moderador capaz de conseguir a participação e o ponto de vista de todos e de cada um. O moderador de grupo deve facilitar a interação grupal, enquanto um observador é encarregado de captar as informações não verbais e, ao final da atividade, auxiliar o moderador a analisar os possíveis vieses ocasionados por problemas decorrentes de sua forma de coordenar a sessão (Westphal, Bógus \& Faria,1996).

Iniciou-se o procedimento com a assinatura do termo de consentimento das participantes, que foi lido previamente pelo moderador; a entrevista foi realizada em um ambiente neutro (sala de reuniões) e a técnica utilizada foi a gravação e filmagem do grupo focal pela auxiliar da pesquisa, seguindo as normas propostas na dissertação de Mestrado de Zimmerman, (2008). 
O moderador fez a leitura das perguntas norteadoras para as residentes, estabelecendo a discussão e fazendo com que houvesse a participação efetiva de todas, com um debate de aproximadamente uma hora, seguido pelas perguntas finais. O resumo dos tópicos pelo moderador ocorreu no final do grupo, que teve a duração de 112 minutos.

Após a realização do grupo focal, as falas foram transcritas por uma profissional da área e análise de conteúdo foi utilizada a técnica de Bardin (2016), sendo realizada de acordo com as fases propostas pela autora, que são: a pré-análise; a exploração do material; o tratamento dos resultados; a inferência e a interpretação.

Sabe-se que a análise categorial pretende levar em consideração a totalidade do texto, classificando- o segundo a frequência dos itens de sentido e são como "gavetas" que carregam elementos de significação contidos nas mensagens, segundo Bardin (2016).

Assim, as categorias que emergiram das falas dos sujeitos da pesquisa com maior constância foram: homossexualidade, formação acadêmica e Residência médica.

O moderador questionou as residentes, se elas haviam tido contato durante as atividades da Residência com pacientes homossexuais, apenas três residentes afirmaram positivamente e relataram suas experiências.

Uma residente destacou o sentimento de solidariedade entre duas mulheres e relatou que:

"eu tive um contato apenas que foi inclusive de um parto, de uma gestação de um casal homoafetivo, que procurou a clínica de reprodução para fazer inseminação para ter um filho, duas mulheres, e eu fiz o parto e a experiência assim... foi boa. Eu vendo o amor das duas, assim, a paciência de uma com a outra, então acho que tudo isso conta bastante, não só no relacionamento homossexual, mas em qualquer tipo de relacionamento, vendo que uma estava lá ajudando a outra, então acho que isso é muito interessante".

Outra residente ficou emocionada diante de um parto com um casal homoafetivo e referiu ter participado de um parto:

"foi um casal de... foram duas mulheres homoafetivas, que fizeram... tiveram essa gravidez a partir dessa fertilização in vitro, e que minha experiência também foi bem legal, porque eu vivenciei um amor bem bonito entre elas, aquele amor, aquela ansiedade pela chegada daquele bebê, uma dando apoio pra outra, e quando o bebê nasceu, aquela emoção, foi bem legal. Eu achei uma coisa interessante, diferente do que eu vejo na maioria dos casos, onde tem uma gestação de casal heterossexual".

A última residente apresentou-se surpresa com a descoberta a relação homossexual entre uma paciente e sua acompanhante, a partir de uma experiência na enfermaria durante a alta de uma paciente:

"Tinha uma paciente que era homossexual, a gente foi passar a história dela para ela tomar um contraceptivo e ela não tinha interesse em contracepção e a gente ficou até com olho aberto "por que que não tem interesse em contracepção?" Depois descobriram que a paciente era homossexual.

Em relação aos programas de Residência Médica, segundo Moll et al, (2014), tem-se que a maioria deles não apresentou tópicos curriculares específicos na abordagem de temas relacionados à saúde de lésbicas, gays, bissexuais e transexuais (LGBT), embora seja necessária a inclusão desses tópicos para melhor atender os pacientes LGBT (Moll et al., 2014).

\section{Resultados e Conclusões}

Os resultados desta pesquisa corroboram com outros pesquisadores, como Hirschtritt et al (2019), que sugeriram a necessidade do desenvolvimento e implementação de currículos educacionais específicos para LGBT para uso em programas de psiquiatria para adultos nos Estados Unidos da América. 
Além disso, pesquisas futuras podem explorar maneiras eficazes de os programas recrutarem, reterem e apoiarem o corpo docente com conhecimentos específicos de LGBT (Hirschtritt et al., 2019).

Diante dessas mudanças do conteúdo baseado na matriz de competência, deve-se propor a execução desses novos paradigmas nos programas de Residência para serem alcançadas as metas definidas pela FEBRASGO, como pode ser exemplificado pela fala de uma residente que sugeriu dinâmicas que poderiam ser usadas para o aprendizado na Residência:

"E enquanto abordagem profissional, eu acho que é muito rico a troca de experiências, eu acho que a gente tem muitos recursos na educação médica que poderiam ter sido aplicados para esse fim mas que não foram, por exemplo, consultas, vídeo-gravação de consultas, a pessoa gravar uma consulta com um casal homoafetivo e depois a gente abrir essa consulta em grupo, de forma, claro, eticamente consentida e, tudo mais, discutir feedbacks, como a pessoa agiu ali, como não agiu, discussão de caso clínico, e também se abrir, perguntar abertamente para as pessoas, perguntar se tem alguma dúvida em relação a relação sexual, construir esse respeito dentro de nós mesmos, se não, se a gente não construir a gente não vai conseguir trazer isso pra nossa prática, é o que eu acho assim, basicamente."

A utilização da técnica do grupo focal para a discussão de um tema polêmico, a partir do seguimento de um rigor metodológico promoveu a participação das residentes e o interesse pelo tópico. Assim, a função do pesquisador foi buscar a interação entre as participantes da pesquisa, para alcançar o objetivo.

A partir do grupo focal, pode-se perceber que apenas três residentes, do total de nove, tiveram vivências no atendimento de pacientes homossexuais e suscitou na pesquisadora a importância do preparo das futuras profissionais no atendimento a esse grupo, como forma de manter a integralidade no cuidado e contemplar a matriz de competência proposta pelo órgão regulador da classe de acordo com as normas vigentes no país. Desse modo, em 2020, foi acrescida, à programação teórica das residentes, uma aula sobre a saúde da mulher bi, trans e lésbica.

\section{Referências}

Bardin, L. (2016). Análise de conteúdo. Tradução: Luís Antero Reto e Augusto Pinheiro. São Paulo: Edições 70, 2016.

Borges, C. D., \& Santos, M. A. dos (2005). Aplicações da técnica do grupo focal: fundamentos metodológicos, potencialidades e limites. Revista da SPAGESP, 6(1), 74-80. Recuperado em 06 de março de 2021, de http://pepsic.bvsalud.org/scielo.php?script=sci_arttext\&pid=S167729702005000100010\&lng=pt\&tlng=pt.

Brasil, C. C. P. (2020). Sentidos atribuídos à sobrecarga de trabalho por residentes de ginecologia e obstetrícia e sua influência no profissionalismo médico. New Trends in Qualitative Research, 3, 895-908. https://doi.org/10.36367/ntqr.3.2020.895-908. v. 3 (2020): Investigação Qualitativa em Saúde: avanços e desafios.

Dall'agnol CM, Magalhães AMM, Mano GCM, Olschowsky A, Silva FP. (2012). A noção de tarefa nos grupos focais. Rev Gaúcha Enferm[Internet]. 2012[cited 2016 Feb 23];33(1):186-90. Available from: http://www.scielo.br/pdf/rgenf/v33n1/a24v33n1.pdf.

Federação Brasileira de Ginecologia e Obstetrícia. FEBRASGO (2019). Matriz de competências em Ginecologia e Obstetrícia Versão 2 - 04/19. Recuperado em 05 de março de 2021.

Feitosa, E. S., Catrib, A. M. F., Brilhante, A. V. M., Moura, S. B. M. e H., Cunha, S. de M., \& Brasil, C. C. P. (2020). Sentidos atribuídos à sobrecarga de trabalho por residentes de ginecologia e obstetrícia e sua influência no profissionalismo médico. New Trends in Qualitative Research, 3, 895-908. https://doi.org/10.36367/ntqr.3.2020.895-908. v. 3 (2020): Investigação Qualitativa em Saúde: avanços e desafios. 
Hirschtritt, M. E. et al (2019). LGBT- Specific Education in General Psychiatry Residency Programs: a Survey of Program Directors. Academic psychiatry, Washington, v. 43, n. 1, p. 41-45, Feb 2019. doi: 10.1007/s40596-018-1008-1.

Júnior, R.P. (2007). Ensino da cirurgia ginecológica nos programas de residência médica do Brasil - Revista Brasileira de Ginecologia e Obstetrícia - Print version ISSN 0100-7203Online version ISSN 1806-9339 - Rev. Bras. Ginecol. Obstet. vol.29 no.2 Rio de Janeiro Feb. 2007 -http://dx.doi.org/10.1590/S0100-72032007000200001.

Long, D.M. (2000). Competency-based residency training: the next advance in graduate medical education. Acad Med. 2000;75(12):1178-83.

Minayo, M.C.S.O. (2014). O desafio do conhecimento. Pesquisa qualitativa em saúde. 14ª edição Hucitec Editora. São Paulo.

Moll, J. et al. (2014). The prevalence of lesbian, gay, bisexual, and transgender health education and training in emergency medicine residency programs: what do we know? Academic emergency medicine, Philadelphia, v. 21, n. 5, p. 608-611, May 2014. doi: 10.1111/acem.12368.

Romão, G.S., Reis, F.J.C., Cavalli, R.C, \& Silva-de-Sá, M.F. (2017). Matriz de Competência em Ginecologia e Obstetrícia: um novo referencial para os programas de residência médica no Brasil. Femina; 45: 173-177.

Sá, M. F.S., \& Romão, G. S. (2019). The Need for a Competence Matrix in Delivery Rooms for the Proper Work of Multiprofessional Teams. Revista Brasileira de Ginecologia e Obstetrícia, 41(1), 1-3. Epub April 15, 2019.https://doi.org/10.1055/s-0039-1677882.

Westphal, M. F., Bógus, C. M., \& Faria, M. M. (1996). Grupos focais: experiências precursoras em programas educativos em saúde no Brasil. Boletim Oficina Sanitária Panamericana, v. 120, n. 6 , p. $472-482$.

Zimmermann, M. H., \& Martins, P. L. O. (2008). Grupo focal na pesquisa qualitativa: relato de experiência. Disponível em: https://educere.bruc.com.br/arquivo/pdf2008/211_86.pdf. Acesso em: $1^{\circ}$ jul. 2019. 